\title{
The Opening of EUV Loops
}

\author{
J. Zhang \& J. Wang \\ Beijing Astronomical Observatory, National Astronomical \\ Observatories, Chinese Academy of Sciences, Beijing 100012, China
}

\begin{abstract}
We analyzed simultaneous data from Huairou Solar Observing Station (HSOS) and the Transition Region and Coronal Explorer (TRACE) of November 4, 1998 in the active region NOAA 8375. The magnetic fields in this region had the following properties: a main positive polarity line-of-sight field was surrounded by relatively weak negative polarity fields; many moving magnetic features emerged from the positive magnetic field, and cancelled (merged) with the opposite (the same) polarity fields. There was a filament on the neutral line between the main positive polarity field and its surrounding negative polarity fields. The filament was cut off at a magnetic cancellation site and separated into two segments. One segment erupted and disappeared; the other segment rose from one end, but another end was fixed at an interface of a pair of opposite magnetic polarities, finally the morphology of this segment appeared as an $\mathrm{H} \alpha$ surge. Comparing $\mathrm{H} \alpha$ filtergrams with EUV images, we noticed that the $\mathrm{H} \alpha$ surge was co-spatial with an EUV surge. The EUV surge was whiplike in shape, then sidewards moved. We suggest that the evolution of the EUV surge represent the process of EUV loop opening.
\end{abstract}

\section{Introduction}

It is commonly accepted that magnetic changes are the cause of solar active phenomena such as flare onsets and filament eruptions. However, magnetic fields can not be reliably observed at chromosphere and corona layers where many kind of active phenomena take place. Recently, space observations have revealed that magnetic loops are a basic component of the magnetized outer solar atmosphere (Acton et al., 1992; Aschwanden et al., 1999), and the active region loops are found to be highly dynamics (Bray et al., 1991; Brekke, Kjeldseth-Moe \& Harrison, 1997). Daily images of the Sun and the corona are available from several experiments such as Yohkoh Soft X-ray Telescope (SXT), the Extreme-ultraviolet Imaging Telescope (EIT) on the Solar and Heliospheric Observatory (SOHO), and the Transition Region and Coronal Explorer (TRACE). They clearly show different kinds of loops all over the solar surface at transition region and coronal temperatures. Therefore, investigating magnetic loops is essential to understand the magnetic configuration in the Sun's outer atmosphere.

Based on the observations of Huairou Solar Observing Station (HSOS) and TRACE, we present in this paper the study of photospheric (chromospheric) 
active phenomena, e.g., magnetic field interaction, filament eruption, and transition region and coronal dynamics.

\subsection{Results}

The general appearance of active phenomena of the active region, NOAA 8375 , is shown in Figure 1. The left column is TRACE $171 \AA$ images. The enhanced emission is presented by darker color. Dot curve ' 1 ' indicates a EUV channel. It was found that the hot material moved from the lower end of the channel and along the channel during the observational interval (ten hours). Comparing with $\mathrm{H} \alpha$ filtergrams (the middle column), we noticed that the channel was cospatial with a $\mathrm{H} \alpha$ filament. Inside the channel, a small EUV loop (indicated by curve '2') existed for several ten minutes. The arrow at 03:31 UT presented an large EUV surge. After the appearance of the EUV surge, the small EUV loop disappeared. This may indicate that the small loop opened. The EUV surge was co-spatial with an $\mathrm{H} \alpha$ surge (shown by an arrow at the $\mathrm{H} \alpha$ 03:29 UT filtergram). At first, the EUV surge was whiplike in shape, then sidewards moved (see the two arrows at EUV 03:42 UT image and at $\mathrm{H} \alpha$ 03:43 UT filtergram). If the material of the EUV surge moved along a loop, the loop should be expanded (or partial opened). From $\mathrm{H} \alpha$ filtergrams, we found that, before the disappearance of the filament, there was a broken point (the arrow at 02:22 UT) on the middle part of the filament. The filament split into two segments from the broken point. One segment erupted and disappeared. The other segment rose from one end, but another end was fixed, finally the morphology of this segment appeared as an $\mathrm{H} \alpha$ surge.

To find the relationship between the magnetic field evolution and the chromosphere corona active phenomena, we present time sequence of line-of-sight magnetograms from HSOS in the right column in Figure 1. White patches present positive polarity fields, and black patches, negative polarity fields. In this active region, many moving magnetic features (Harvey and Harvey, 1973) emerged near the boundary of the main positive polarity magnetic patch and moved radially outward, they cancelled (merged) with the opposite (same) polarity magnetic fields. Two square brackets at 00:39 UT marked two cancellation magnetic flux patches. The filament was cut off at the cancellation site. A negative magnetic patch (marked by two brackets on the lower-left corner at 00:39 UT) cancelled with its peripheral positive polarity magnetic fields, and thus caused in the appearance of the $\mathrm{H} \alpha$ and TRACE EUV surge.

Acknowledgments. This work is supported by the Major Project 19791090 , funded by the National Natural Science Foundation of China (NSFC). The authors are indebted to the Huairou staff for obtaining good observations. They are grateful to all members of $T R A C E$ team for providing the wonderful data.

\section{References}

Acton, L. W., Feldman, U., Bruner, M. E., Doschek, G. A., Hirayama, T., Hudson, H. S., Lemen, J. R., Ogawara, Y. T., \& Tsuneta, S. 1992, PASJ, 44, L71 


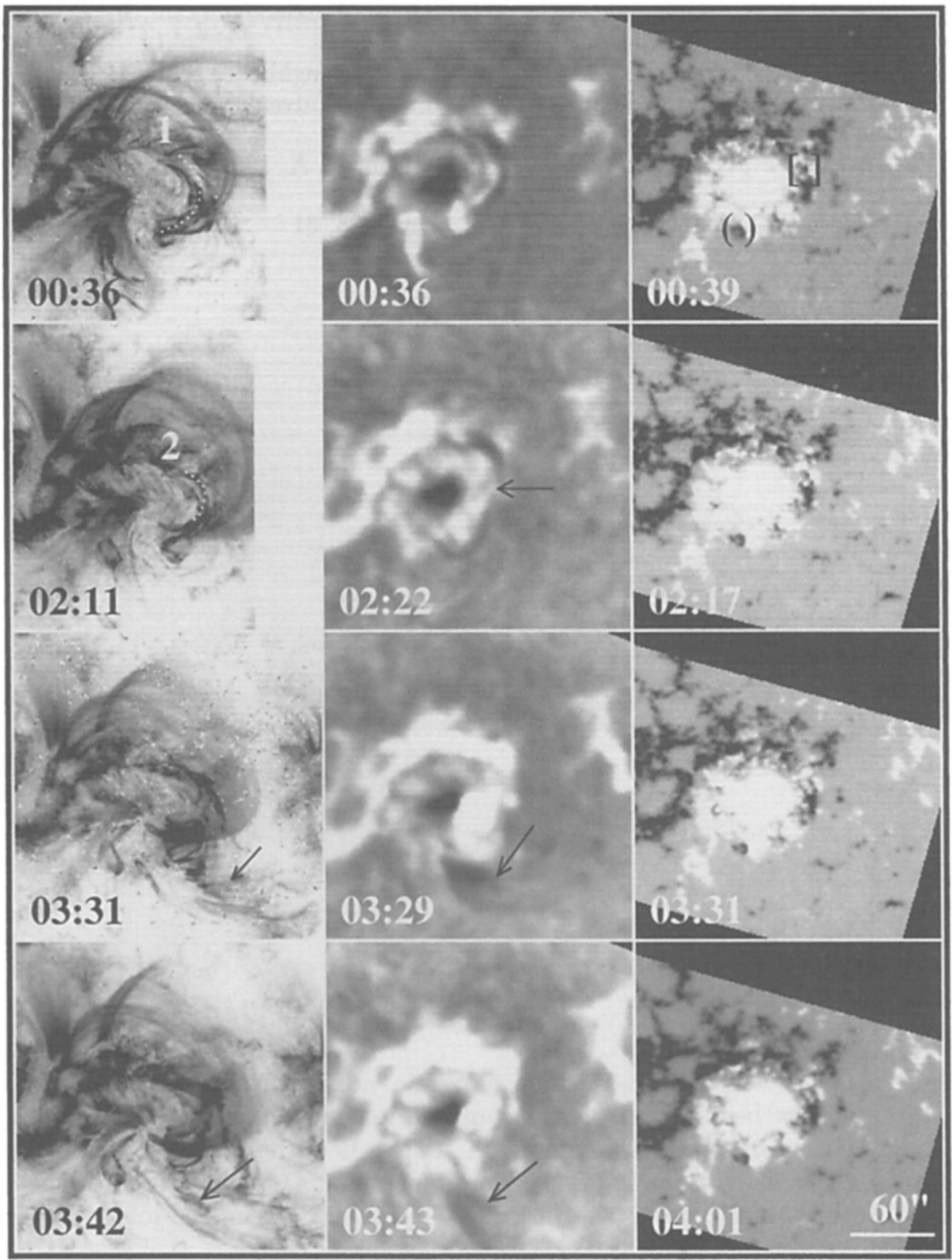

Figure 1. Time sequences of EUV $171 \AA$ images (the left column), $\mathrm{H} \alpha$ filtergrams (the middle column) and line-of-sight magnetograms (the right column) showing the chromosphere-corona active phenomena in the active region NOAA 8375 . 
Aschwanden, M. J., Newmark, J. S., Delaboudinierem J. -P., Neupett, W. N., Klimchuk, J. A., Gary, G. A., Portier-Fonzzani, F., \& Zucker, A. 1999, ApJ, 515, 842

Bray, R. J., Cram, L. E., Durrant, C. J. \& Loughhead, R. E. 1991, Plasma loops in the Solar Corona, Cambridge University Press, Cambridge

Brekke, P., Kjeldseth-Moe, O., \& Harrison, R. A. 1997, Sol. Phys., 175,511

Harvey, K., \& Harvey, J. 1973, Sol. Phys., 28, 61 